\title{
Application of a high resolution T1 mapping with MOLLI (hrMOLLI) in patients in clinical setting: a reproducibility study
}

\author{
Ana Pastor ${ }^{1}$, Zhong Chen ${ }^{1}$, Tobias Voigt ${ }^{2}$, Tobias Schaeffter ${ }^{1}$, Reza Razavi ${ }^{1}$, Eike Nagel ${ }^{1}$, Valentina O Puntmann ${ }^{1 *}$ \\ From 15th Annual SCMR Scientific Sessions \\ Orlando, FL, USA. 2-5 February 2012
}

\section{Summary}

Several methodologies were proposed to evaluate myocardial $\mathrm{T} 1$ values by cardiovascular magnetic resonance based on Gadolinium contrast enhancement. Whereas potentially valuable for evaluation of myocardial fibrosis, none of these methodologies have been sufficiently robust and consistently translated into clinical routine. We propose that imaging with a high-resolution modified Look-Locker inversion recovery sequence (hrMOLLI) of native and post-contrast myocardium can provide a robust tool in the clinical setting.

\section{Background}

Myocardial fibrosis is associated with myocardial dysfunction and remodelling leading to adverse outcomes. We propose that imaging with a high-resolution modified Look-Locker inversion recovery sequence (hrMOLLI) of native and post-contrast myocardium can provide a robust tool in the clinical setting.

\section{Methods}

Fifty subjects (age $47 \pm 7.4$ years) underwent a routine CMR protocol on $3 \mathrm{~T}$ clinical scanner with dual 32 channel coil and multi-transmit system. A single breathhold hrMOLLI imaging was performed in an equatorial short axis slice prior and at selected intervals following administration of $0.2 \mathrm{mmol} / \mathrm{kg}$ gadolinium-DTPA contrast (10, 20, 40 minutes) (Figure1). Imaging parameters were FOV 320x320; TR/TE $3.2 / 1.57 \mathrm{~ms}$, flip angle $50^{\circ}$, interpolated voxel size $0.9 \times 0.9 \times 8 \mathrm{~mm}$, phase encoding steps $n=166$, Trigger delay: $450 \mathrm{msec}$. T1 map values were obtained by 3 independent observers by placing

'Cardiovascular Imaging, King's College London, London, UK

Full list of author information is available at the end of the article the region of interest (ROI) within the septal myocardium (Figure 2). In patients with myocardial scar on late gadolinium enhancement, ROIs were derived from the remote areas only. A subset of healthy subjects $(n=6)$ had a repeated native hrMOLLI imaging for interstudy reproducibility within 3 weeks of the original scan. Agreement between and within observers was examined by paired t-test and linear regressions.

\section{Results}

T1 maps could be analysed in $94 \%$ of all images obtained. Intra-observer mean differences (MD) for T1 values were $1.3 \mathrm{~ms}$ (95\%CI:-12.4-15.4) for native scans and $0.7 \mathrm{~ms}(-7.9-12.3)$ for overall postcontrast scans, whereas interobserver MDs were $0.3 \mathrm{~ms}(-6.3-5.3)$ and 0.1 (-3.4 to 4.2$)$, respectively. We further demonstrate an excellent overall (pre- and postcontrast) intra-and interobserver agreement in T1 relaxation times (intraobserver: $r=0.94, p<0.0001$; interobserver: $R=0.91$, $\mathrm{p}<0.0001)$. When assessed separately the postcontrast data showed better agreement within and between observers, respectively $(\mathrm{r} \sim 0.94, \mathrm{p}<0.0001$ for both) than in the precontrast native scans (intraobserver $\mathrm{r}=0.81, \mathrm{p}$ $<0.0001$; interobserver $\mathrm{r}=0.79, \mathrm{p}<0.001)$. We further revealed close agreements between repeated studies $(\mathrm{r}=0.91, \mathrm{p}<0.001 ; \mathrm{MD}: 2.4 \mathrm{msec}$ (95\%CI: $-3.1-10.2)$.

\section{Conclusions}

We demonstrate that imaging with hrMOLLI as a single breath-held scan pre and postcontrast administration provides a robust tool to reproducibly derive T1 relaxation times in clinical setting.

\section{Funding}

NIHR British Research Centre. 


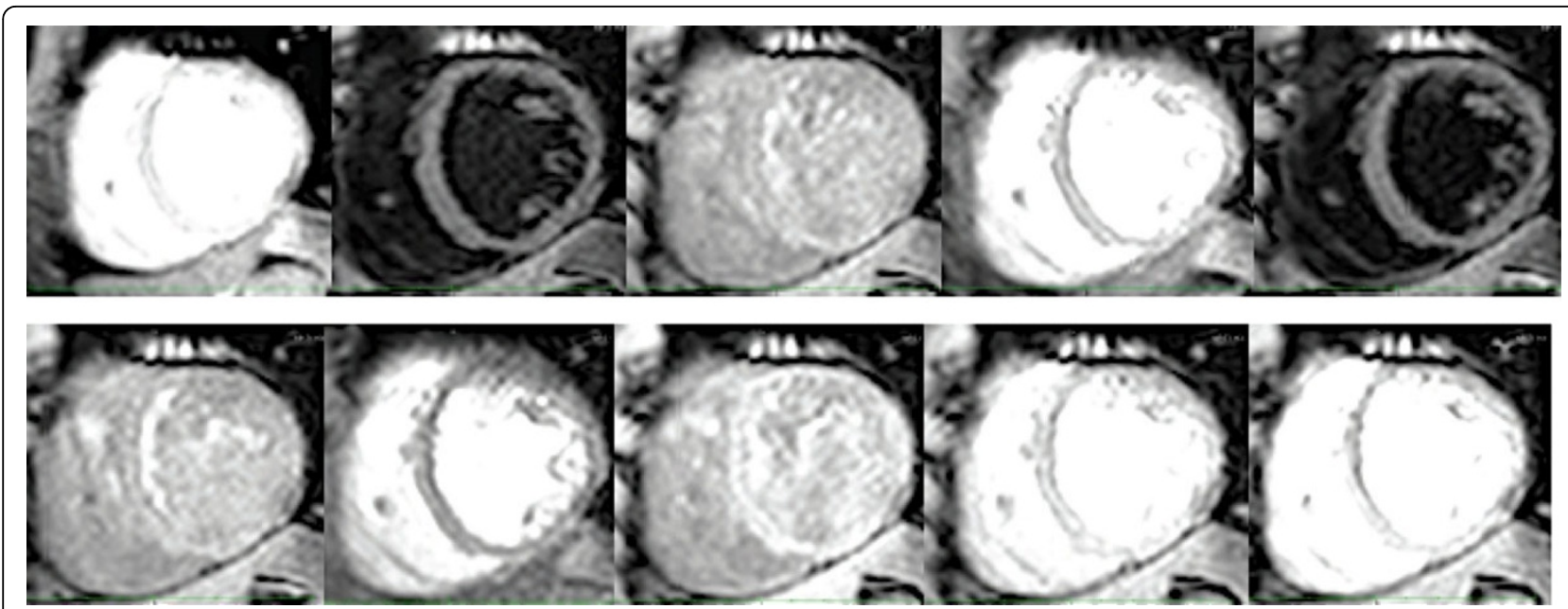

Figure 1 Representative images of post-contrast MOLLI sequence (11th phase omitted).

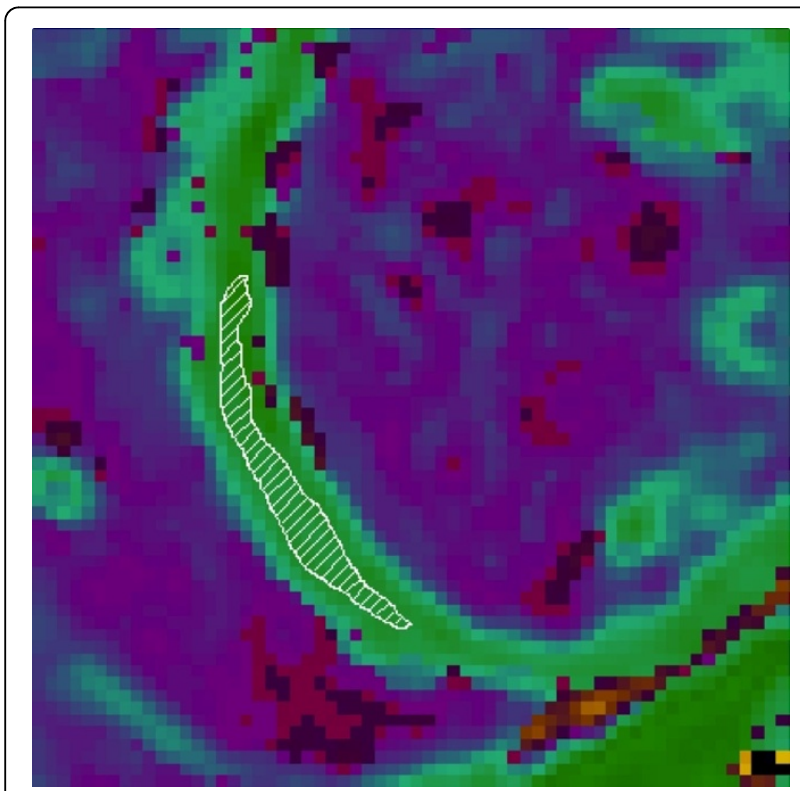

Figure 2 T1 map with outline of region of interest placed conservatively within the septal myocardium.

\section{Author details}

${ }^{1}$ Cardiovascular Imaging, King's College London, London, UK. ${ }^{2}$ Philips Healthcare, London, UK.

Published: 1 February 2012

doi:10.1186/1532-429X-14-S1-082

Cite this article as: Pastor et al: Application of a high resolution T1 mapping with MOLLI (hrMOLLI) in patients in clinical setting: a reproducibility study. Journal of Cardiovascular Magnetic Resonance 2012 14(Suppl 1):082.

\section{Submit your next manuscript to BioMed Central} and take full advantage of:

- Convenient online submission

- Thorough peer review

- No space constraints or color figure charges

- Immediate publication on acceptance

- Inclusion in PubMed, CAS, Scopus and Google Scholar

- Research which is freely available for redistribution 